\title{
Extended Fluid-Dynamic Model for Micron-Scale Flows Based on Gaussian Moment Closure
}

\author{
J. G. MDonald* and C. P. T. Groth ${ }^{\dagger}$ \\ University of Toronto Institute for Aerospace Studies \\ 4925 Dufferin Street, Toronto, Ontario, Canada, M3H 5 T6
}

\begin{abstract}
An extended fluid-dynamic model for micron-scale flows with heat transfer based on the Gaussian moment closure is presented. The standard Gaussian closue moment equations are hyperbolic and closure is achieved by assuming a form for the velocity distribution function which will, by construction, yield zero heat flux; the proposed extension leads to the addition of anisotropic thermal-diffusion terms having an elliptic nature. The extended model can be achieved by taking a Chapman-Enskog-type expansion about either the Gaussian moment equations of the kinetic equation and both methods are presented here. The elliptic nature of the additional generalized heat-flux terms lead to fully dispersed shock wave solutions, unlike the partially dispersed solutions provided by the Gaussian closure as the Mach number increases. Using a Godunov-type finite-volume scheme with block based adaptive-mesh refinement (AMR) on body-fitted multi-block meshes, the proposed equations are applied to several canonical continuum and micron-scale flow situations such as shock structure, Couette flow, and flow past a circular cylinder, as well as for transonic flow past a micro airfoil. Comparisons with analytic, experimental, and direct-simulation Monte Carlo (DSMC) results are made and demonstrate the capabilities of the proposed non-equilibrium model.
\end{abstract}

\section{Introduction and Background}

Non-equilibrium micron-scale flows possess a variety of characteristics which make them difficult to simulate by means of existing numerical techniques. Flow Knudsen numbers, $\mathrm{Kn}$, in the range $0.01<\mathrm{Kn}<10$ may be encountered, even for pressures above one atmosphere, and, as a result, non-continuum and thermal non-equilibrium effects can significantly influence momentum and heat-transfer phenomena in typical microchannel flows. ${ }^{1,2}$ In most cases, these flows are in the subsonic to low-supersonic regimes and, due to their micro-geometries, have low Reynolds numbers and remain laminar. These flows can deviate significantly from local thermodynamic equilibrium, and thus, traditional equation sets, such as the Euler and Navier-Stokes equations cannot be used to model flow behaviour.

Particle-simulation techniques, such as the direct-simulation Monte Carlo (DSMC) method, ${ }^{3}$ have been developed for the prediction of general non-equilibrium gaseous flows. However, for near-continuum through to transitional-regime flows, the computational costs incurred by these techniques are considerable. This is especially true for flows with low Mach numbers and, in these situations, computational expense can prohibit their usage. ${ }^{4,5}$

Alternate approaches for the simulation of micron-scale flows include methods based on moment closures. ${ }^{6,7}$ These methods are based on approximate solutions to the Boltzmann equation of kinetic theory. In these techniques, an assumed form for the probability distribution function is chosen such that moments (or macroscopic quantities), whose transport can then be determined by a set of moment equations, can be determined. Moment closures provide an extended set of partial differential equations (PDEs) describing the transport of macroscopic fluid properties. In general, the solution of these PDEs require considerably less effort than that associated with obtaining solutions using particle simulation methods. Previous studies by the authors have demonstrated the applicability of moment-closure based techniques to micron-scale flows. ${ }^{8}$

*PhD Candidate, Email: mcdonald@utias.utoronto.ca, Student Member AIAA.

${ }^{\dagger}$ Associate Professor, Email: groth@utias.utoronto.ca, Senior Member AIAA. 
Moment closures, unfortunately, are not without their limitations. Early hierarchies of closures, such as those proposed by Grad, ${ }^{6}$ provide hyperbolic PDEs for the time evolution of non-equilibrium quantities. The equations, however, suffer from closure breakdown and loss of hyperbolicity for what appear to be relatively benign flows. The Grad closures also do not guarantee moment realizability of solution』 The hyperbolicity of moment equations, though pleasing from a physical point of view ${ }^{b}$, also leads to a well known problem of discontinuous, or partially dispersed, shock-wave solutions which contain sub-shocks when the upstream flow exceeds a critical speed. These sub-shocks are an aphysical artifact of the moment closures.

Levermore has proposed a new hierarchy of non-perturbative moment closures with several desirable mathematical properties. ${ }^{7}$ Closures belonging to this hierarchy do not suffer from the closure-breakdown deficiency of many previous closures and the resulting PDEs remain hyperbolic with realizable moment solutions for all physically realistic situations. Unfortunately, moments of all but the two lowest-order members of this hierarchy prove impossible to evaluate analytically, and thus, explicit forms of moment equations with all solution fluxes expressed explicitly in terms fo the lower-order moments cannot be determined. The two closures which can be evaluated are the so-called five- and ten-moment closures which result in the Euler equations, in the prior case, and the Gaussian closure, in the latter. In addition to the typical continuity and momentum equations, the Gaussian closure furnishes a set of equations for an anisotropic pressure tensor. However, it is deficient in that, by construction, it always results in a zero heat-flux vector and tensor. This closure is therefore obviously unsuitable for situations in which heat flux plays a significant role. Higher-order closures of the Levermore variety would provide moment equations for heat-transfer phenomena, however, as stated earlier, explicit analytic forms of the transport equations for the moments of interest cannot be determined.

In an effort to extend the Gaussian closure to include higher-order effects, Groth et al. ${ }^{9,10}$ formulated perturbative variants to the original moment closure yielding a new extended fluid dynamic model. The most well studied of these closures is a 35-moment closure. This closure remains hyperbolic for a large range of flow situations, however it still does not possess the robustness of the original Levermore closures. Recently, Stuchtrup ${ }^{11}$ has shown a technique for the regularization of moment equations and has applied this to the 13-moment equations of Grad. In essence, this technique consists of creating an expansion about the moment equations, thus allowing small deviations from the assumed distribution function. A required assumption for this procedure is that deviations from the assumed distribution function are suppressed by inter-molecular collisions more quickly than non-equilibrium effects contained in the original moment equations. In this paper, a similar regularization technique is applied to the Gaussian closure. This procedure results in the addition of elliptic terms to the standard equations of the Gaussian closure. The resulting terms lead to a heat-transfer treatment which is dependent on the anisotropic pressure tensor. The elliptic nature of the added terms leads to smooth solutions, even through high-Mach-number shocks.

The following section will describe the derivation of the present correction to the Gaussian equations. This is done both by expansion about the moment equations (this is the technique used by Struchtrup), and also by explicitly taking an expansion around the assumed form of the distribution function in the kinetic equation. It is shown that both techniques yield identical results. Following these derivations, the application of a finite-volume scheme to the solution of the resulting equations is detailed. Numerical results for a range of canonical continuum and micron-scale flow problems are then explored, including shock structure, Couette flow, and flow past a circular cylinder, as well as transonic flow past a micro airfoil. Comparisons with analytic, experimental, and direct-simulation Monte Carlo (DSMC) results are also made and demonstrate the capabilities of the proposed non-equilibrium model. Finally, some conclusions regarding the applicability of these equations to practical micron-scale applications are drawn.

\section{Governing Equations}

\section{II.A. Gaskinetic Theory}

Moment closures arise from the field of gaskinetic theory. This theoretical approach takes into account the particle nature of gases by defining a probability density function, $\mathcal{F}\left(x_{i}, v_{i}, t\right)$, in six-dimensional phase space which specifies the probability of finding particles at a given location, $x_{i}$, and time, $t$, having a particular gas

\footnotetext{
${ }^{a}$ Moment realizability refers to the existence of a physically realistic velocity distribution function which corresponds to the predicted moment values.

${ }^{\mathrm{b}}$ Hyperbolic equations predict finite speed of propagation for infinitesimal disturbances. This is in contrast to elliptic equations which predict infinite speeds of propagation.
} 
velocity, $v_{i}$. Macroscopic "observable" properties of the gas are then obtained by taking appropriate velocity moments of $\mathcal{F}$. This is done by integrating the product of the distribution function and an appropriate velocity dependent weight over all velocity space,

$$
\left\langle M\left(v_{i}\right) \mathcal{F}\right\rangle=\int_{-\infty}^{\infty} M\left(v_{i}\right) \mathcal{F}\left(x_{i}, v_{i}, t\right) d^{3} v
$$

The evolution of the velocity distribution function is prescribed by the Boltzmann equation. ${ }^{12-14}$ This is an integro-differential equation for $\mathcal{F}$ having the form:

$$
\frac{\partial \mathcal{F}}{\partial t}+v_{i} \frac{\partial \mathcal{F}}{\partial x_{i}}+a_{i} \frac{\partial \mathcal{F}}{\partial v_{i}}=\delta \mathcal{F}
$$

Here $a_{i}$ is the acceleration due to external forces. The term on the right hand side of the equation, $\delta \mathcal{F}$, is the Boltzmann collision integral and represents the time rate of change of the distribution function produced by inter-particle collisions.

Transport equations governing the time evolution of macroscopic quantities can be derived by evaluating velocity moments of the Boltzmann equation given above. This leads to the so-called Maxwell's equation of change. The transport equations describing the evolution of the moment $\langle M \mathcal{F}\rangle$ is then given by

$$
\frac{\partial}{\partial t}\langle M \mathcal{F}\rangle+\frac{\partial}{\partial x_{i}}\left\langle v_{i} M \mathcal{F}\right\rangle=\Delta\langle M \mathcal{F}\rangle .
$$

Here the acceleration field is taken to be zero (as will be the case throughout the present work), $\Delta\langle M \mathcal{F}\rangle=$ $\langle M \delta F\rangle$ is the effect of collisions on the moment quantity, and $M$ is an appropriate velocity dependent weight.

It is at this point that the problem of closure becomes apparent. The time evolution of a moment $\langle M \mathcal{F}\rangle$ is clearly dependent on the spacial divergence of $\left\langle v_{i} M \mathcal{F}\right\rangle$, a moment of one higher order in terms of the velocity, $v_{i}$. This pattern is repeat with the time evolution of every moment being dependent on a moment of one higher order in $v_{i}$. In general, an infinite number of moment equations is required to fully describe the evolution of a macroscopic flow quantity, and solving this infinite system is equivalent to solving Eqn (2).

One technique used to obtain moment closure is to restrict the distribution function to an assumed form. ${ }^{6}$ Restricting the form of the distribution function has the effect of restricting the value of certain higher-order moments to be functions of lower-order moments, thus furnishing a closing relationship in the moment equations. For example, for a monatomic gas if the distribution function is assumed to have the form

$$
\mathcal{F}\left(x_{i}, v_{i}, t\right)=\mathcal{M}\left(x_{i}, v_{i}, t\right)=n\left(\frac{\beta}{\pi}\right)^{\left(\frac{3}{2}\right)} e^{\left(-\beta c_{i} c_{i}\right)},
$$

where $n$ is the number density, $c_{i}$ is the random component of the particle velocity and $\beta$ is a function of the local temperature, the method of moments will lead to the well-known compressible Euler equations describing the time evolution of density, momentum, and energy. Equation (4) is the well-known the MaxwellBoltzmann distribution, $\mathcal{M}$, describing the equilibrium behaviour of a monatomic gas. It can be shown that the collision operator will force all distribution functions towards this form, and, once in this state, the collision operator will produce no further effects. This entropy property of the collision operator is well established by Boltzmann's H theorem. Gases described by this distribution function are said to be in local thermodynamic equilibrium.

\section{II.B. The Collision Operator}

In general, the collision operator present in Eqns (2) and (3) are difficult or impossible to evaluate. Fortunately, for many engineering problems, approximate collision terms prove adequate. The most commonly used approximation is the BGK collision operator. ${ }^{15}$ This operator can be written as

$$
\delta \mathcal{F}=-\frac{\mathcal{F}-\mathcal{M}}{\tau} .
$$

This is often referred to as the "relaxation-time" collision operator, as it describes the relaxation of a general distribution function $\mathcal{F}$ towards the equilibrium Maxwellian with characteristic time $\tau$. The parameter $\tau$ can be chosen such that the correct continuum viscosity of the gas is predicted ( $\mu=\tau p$, where $\mu$ is the fluid 
viscosity and $p$ is the thermodynamic pressure). This model will also predicts the correct free-molecular behaviour of the collision operator (i.e., it has no effect when $\tau$ approaches infinity) and can be shown to satisfy Boltzmann's H theorem. A well-known limitation of the BGK model, however, is that it always yields a Prandtl number of

$$
\frac{2}{5} \frac{\gamma}{\gamma-1}=\left\{\begin{array}{cl}
1 & \text { for monatomic gases, and } \\
1.4 & \text { for diatomic gases. }
\end{array}\right.
$$

This is in contradiction with physical Prandtl numbers for most gases, which tend to be less than one.

There are several other collision-operator models available which can provide more realistic Prandtl numbers. In the present work, an approximate collision term proposed by Holway ${ }^{16}$ will be used to describe collisional processes for monatomic gases. This model, often referred to the ellipsoidal statistical model, preserves much of the simplicity of the BGK model, while allowing for a tunable Prandtl number. It can be written as

$$
\delta \mathcal{F}=-\frac{\mathcal{F}-\mathcal{G}_{E S}}{\tau_{E S}},
$$

where

$$
\mathcal{G}_{E S}\left(x_{i}, v_{i}, t\right)=\frac{\rho}{m(2 \pi)^{3 / 2}\left(\operatorname{det} T_{\alpha \beta}\right)^{1 / 2}} \exp \left(-\frac{1}{2} T_{i j}^{-1} c_{i} c_{j}\right) .
$$

where $m$ is the gas particle mass and $\rho$ is the mass density. It will be seen in the next sub-section that this distribution is a Gaussian distribution function which possesses a modified pressure tensor. The tensor $T_{i j}$ is defined as

$$
T_{i j}=(1-\nu) R T \delta_{i j}+\nu \Theta_{i j}
$$

where $\Theta_{i j}$ is a symmetric 'temperature' tensor given by $\Theta_{i j}=P_{i j} / \rho$ and $P_{i j}$ is the generalized pressure tensor of the gas. The deviatoric stress tensor, $\tau_{i j}$, is related to the pressure tensor as follows: $\tau_{i j}=\delta_{i j} p-P_{i j}$, where $p=P_{i i} / 3$ is the equilibrium isotropic pressure. If the parameters $\tau_{E S}$ and $\nu$ are chosen such that $(1-\nu) \mu=\tau_{E S} p$ and $(1-\nu) \operatorname{Pr}=1$ the model will predict the correct values for fluid viscosity and thermal conductivity in the continuum limit. The ellipsoidal statistical collision model's adherence to Boltzmann's $\mathrm{H}$ theorem was only recently demonstrated by Andries and Perthame ${ }^{17}$ and only for monatomic gases. It is important to note that the relaxation times for these two models $\left(\tau\right.$ and $\left.\tau_{E S}\right)$ differ by a factor of the Prandtl number and the moment equations which follow will be written in terms of the relaxation time for the standard BGK model for consistency with traditional forms of these equations.

\section{II.C. The Gaussian Closure}

In the Gaussian closure, the velocity distribution function for monatomic gaseous particles is assumed to have the following form:

$$
\mathcal{G}\left(x_{i}, v_{i}, t\right)=\frac{\rho}{m(2 \pi)^{3 / 2}\left(\operatorname{det} \Theta_{\alpha \beta}\right)^{1 / 2}} \exp \left(-\frac{1}{2} \Theta_{i j}^{-1} c_{i} c_{j}\right),
$$

This assumed distribution function provides moment closure as $\left\langle m c_{i} c_{j} c_{k} \mathcal{G}\right\rangle=0$ by construction. Unfortunately, this relationship causes the heat flux vector, $q_{i}=\frac{1}{2}\left\langle m c_{i} c_{j} c_{j} \mathcal{G}\right\rangle$ to also vanish. This demonstrates a significant limitation of the standard Gaussian closure: its inability to account for the effects of thermal diffusion.

The Gaussian distribution appears to have been first derived in early work by Maxwell ${ }^{18}$ and then re-discovered in subsequent but independent research by both Schlüter ${ }^{19,20}$ and Holway. ${ }^{16,21-23}$ It may be regarded as a generalization of the bi- and tri-Maxwellian velocity distribution functions that does not require the identification of the planes of principal stress. ${ }^{19,20,24,25}$ This approximate non-equilibrium distribution possesses a Gaussian-like distribution in each of the principal strain axes, physically, this corresponds to a non-equilibrium gas with a different temperature in each direction.

Substitution of Equation (9) into Equation (3) with use of the approximate relaxation-time collision term of Holway ${ }^{16}$ described above leads to the following set of ten PDEs describing the transport of the macroscopic quantities $\rho, \rho u_{i}$, and $P_{i j}$ :

$$
\frac{\partial \rho}{\partial t}+\frac{\partial \rho u_{k}}{\partial x_{k}}=0
$$




$$
\begin{gathered}
\frac{\partial}{\partial t}\left(\rho u_{i}\right)+\frac{\partial}{\partial x_{k}}\left(\rho u_{i} u_{k}+P_{i k}\right)=0 \\
\frac{\partial P_{i j}}{\partial t}+\frac{\partial}{\partial x_{k}}\left(u_{k} P_{i j}\right)+P_{j k} \frac{\partial u_{i}}{\partial x_{k}}+P_{i k} \frac{\partial u_{j}}{\partial x_{k}}=-\frac{1}{\tau}\left(P_{i j}-\frac{1}{3} P_{k k} \delta_{i j}\right) .
\end{gathered}
$$

The above equations were derived for a monatomic gas with no internal degrees of freedom. A modification must be made to account for energy that can be present due to the extra internal degrees if diatomic molecules are to be considered. Such an extension has been proposed Hittinger ${ }^{26}$ and has been previously studied by the authors. ${ }^{8}$ This extension adds an extra transport equation for internal rotational energy, resulting in the following moment equations:

$$
\begin{gathered}
\frac{\partial \rho}{\partial t}+\frac{\partial \rho u_{k}}{\partial x_{k}}=0 \\
\frac{\partial}{\partial t}\left(\rho u_{i}\right)+\frac{\partial}{\partial x_{k}}\left(\rho u_{i} u_{k}+P_{i k}\right)=0 \\
\frac{\partial P_{i j}}{\partial t}+\frac{\partial}{\partial x_{k}}\left(u_{k} P_{i j}\right)+P_{j k} \frac{\partial u_{i}}{\partial x_{k}}+P_{i k} \frac{\partial u_{j}}{\partial x_{k}}=-\frac{3 P_{i j}-P_{k k} \delta_{i j}}{3 \tau_{t}}-\frac{2\left(P_{k k}-3 E_{r o t}\right)}{15 \tau_{r}} \delta_{i j}, \\
\frac{\partial}{\partial t}\left(E_{r o t}\right)+\frac{\partial}{\partial x_{k}}\left(u_{k} E_{r o t}\right)=-\frac{3 E_{r o t}-P_{k k}}{5 \tau_{r}} .
\end{gathered}
$$

Here, $\tau_{t}$ and $\tau_{r}$ are the relaxation times associated with translational and rotational degrees of freedom respectively.

\section{Extended Fluid Treatment for Thermal-Diffusion Effects}

As stated earlier, one of the major shortfalls of the Gaussian closure is its inability to account for thermal diffusion. This is due to the construction of the assumed form of the distribution function used to obtain moment closure. By allowing small deviations from the Gaussian distribution, however, thermal diffusion can be re-introduced into the moment equations. This can be done by taking an appropriate ChapmanEnskog perturbative expansion of either the moment equations (Eq. 10 12) or the kinetic equation using the Gaussian distribution as the base distribution. Both of these techniques are detailed here.

\section{III.A. Perturbative Expansion About the Moment Equations}

A similar technique was previously used by Struchtrup in the regularization of the 13 -moment equations. ${ }^{11}$ For this expansion, it is convenient to define the quantities

$$
K_{i j k l}=m\left\langle c_{i} c_{j} c_{k} c_{l} \mathcal{F}\right\rangle-m\left\langle c_{i} c_{j} c_{k} c_{l} \mathcal{G}\right\rangle=m\left\langle c_{i} c_{j} c_{k} c_{l} \mathcal{F}\right\rangle-\frac{1}{\rho}\left[P_{i j} P_{k l}+P_{i k} P_{j l}+P_{i l} P_{j k}\right] .
$$

This is the deviation of the fourth random-velocity moment from that calculated using a Gaussian distribution. The general moment quantities are then re-written as a perturbative expansion about their values as predicted by the Gaussian closure. This is done by introducing a formal smallness parameter, $\epsilon$, which is used to scale the solution and moment equations. For example, the scaled solution for the generalized heat flux $Q_{i j k}$ and previously defined $K_{i j k l}$ tensors are written as

$$
Q_{i j k}=Q_{i j k}^{(\mathcal{G})}+\epsilon Q_{i j k}^{(1)}+\epsilon^{2} Q_{i j k}^{(2)}+\epsilon^{3} Q_{i j k}^{(3)}+\cdots,
$$

and

$$
K_{i j k l}=K_{i j k l}^{(\mathcal{G})}+\epsilon K_{i j k l}^{(1)}+\epsilon^{2} K_{i j k l}^{(2)}+\epsilon^{3} K_{i j k l}^{(3)}+\cdots .
$$

Here, the superscript $(\mathcal{G})$ denotes the value for a moment calculated using a Gaussian distribution function and the superscript $(n)$ denotes the $n$-th order correction. The moment equation for the moment $\left\langle c_{i} c_{j} \mathcal{F}\right\rangle$ in its general form (without using an assumed form for the distribution function) is

$$
\frac{\partial P_{i j}}{\partial t}+\frac{\partial}{\partial x_{k}}\left(u_{k} P_{i j}\right)+P_{j k} \frac{\partial u_{i}}{\partial x_{k}}+P_{i k} \frac{\partial u_{j}}{\partial x_{k}}+\frac{\partial Q_{i j k}}{\partial x_{k}}=-\frac{1}{\tau}\left(P_{i j}-\frac{1}{3} P_{k k} \delta_{i j}\right) .
$$


It can be seen that for this general case, there is a term, $\frac{\partial Q_{i j k}}{\partial x_{k}}$ which is not present in Equation 12 . Setting this third-order tensor to be identically zero is how closure was obtained in the Gaussian model. However, in the current technique, a non-zero first-order deviation, i.e., $Q_{i j k}^{(1)}$, is introduced. For small deviations from the Gaussian closure, an approximation to this term can be determined by writing the scaled moment equation for $Q_{i j k}$, which, again using the approximate relaxation-time collision term of Holway, ${ }^{16}$ can be written as

$$
\begin{aligned}
& \frac{\partial Q_{i j k}}{\partial t}+\frac{\partial}{\partial x_{l}}\left(u_{l} Q_{i j k}\right)+Q_{j k l} \frac{\partial u_{i}}{\partial x_{l}}+Q_{i k l} \frac{\partial u_{j}}{\partial x_{l}}+Q_{i j l} \frac{\partial u_{k}}{\partial x_{l}} \\
& +P_{k l} \frac{\partial}{\partial x_{l}}\left(\frac{P_{i j}}{\rho}\right)+P_{j l} \frac{\partial}{\partial x_{l}}\left(\frac{P_{i k}}{\rho}\right)+P_{i l} \frac{\partial}{\partial x_{l}}\left(\frac{P_{j k}}{\rho}\right)+\frac{\partial K_{i j k l}}{\partial x_{l}}=-\frac{\operatorname{Pr}}{\epsilon \tau} Q_{i j k},
\end{aligned}
$$

for a monatomic gas. Note that, in the expression above, Equation (17) has been used and the scaling parameter, $\epsilon$, has been explicitly introduced on the right-hand side. The small parameter $\epsilon$ is introduced in accordance with the assumption that deviations of the moment quantities from those predicted by the Gaussian closure (i.e., $Q_{i j k}=0$ ) will be attenuated rapidly by collisional processes. By making use of equations 18 and 19 , it can be seen that the unscaled zeroth-order terms of Equation (21) yields

$$
Q_{i j k}^{(\mathcal{G})}=0
$$

in agreement with the Gaussian closure. The equation allowing for unscaled first-order deviations becomes

$$
Q_{i j k}^{(1)}=-\frac{\tau}{\operatorname{Pr}}\left[P_{k l} \frac{\partial}{\partial x_{l}}\left(\frac{P_{i j}}{\rho}\right)+P_{j l} \frac{\partial}{\partial x_{l}}\left(\frac{P_{i k}}{\rho}\right)+P_{i l} \frac{\partial}{\partial x_{l}}\left(\frac{P_{j k}}{\rho}\right)\right] .
$$

This is the correction which will be used herein to introduce thermal diffusion to the Gaussian equations.

\section{III.B. Perturbative Expansion About the Kinetic Equation}

The above derivation was based on an expansion about the moment equations. The same result can also be obtained from an expansion about the kinetic equation with the ellipsoidal statistical collision model of Holway. ${ }^{16}$ To do this, it is convenient to write the collision operator in a slightly different form. This is done by first assuming $(1-\nu)=\epsilon \ll 1$ and rewriting the expression for the tensor $T_{i j}$ as

$$
\begin{aligned}
T_{i j} & =(1-\nu) R T \delta_{i j}+\nu \Theta_{i j} \\
& =\epsilon R T \delta_{i j}+(1-\epsilon) \Theta_{i j} \\
& =\Theta_{i j}+\epsilon\left[R T \delta_{i j}-\Theta_{i j}\right] \\
& =\Theta_{i \alpha}\left[\delta_{\alpha j}-\epsilon \Lambda_{\alpha j}\right]
\end{aligned}
$$

where $\Theta_{i \alpha} \Lambda_{\alpha j}=\left[R T \delta_{i j}-\Theta_{i j}\right]$. It can be seen in Equation 77 that $T_{i j}^{-1}$ is needed. This can be written as

$$
T_{i j}^{-1}=\left[\delta_{i \alpha}-\epsilon \Lambda_{i \alpha}\right]^{-1} \Theta_{\alpha j}^{-1}
$$

Provided the spectral radius of $\epsilon \Lambda_{i \alpha}$ is less than unity, a fact that is true given the assumption of the smallness of $\epsilon$, the term $\left[\delta_{i \alpha}-\epsilon \Lambda_{i \alpha}\right]^{-1}$ can be formally expanded as

$$
\left[\delta_{i \alpha}-\epsilon \Lambda_{i \alpha}\right]^{-1}=\delta_{i \alpha}+\epsilon \Lambda_{i \alpha}+\epsilon^{2} \Lambda_{i \alpha}^{2}+\epsilon^{3} \Lambda_{i \alpha}^{3}+\cdots=\sum_{n=0}^{\infty} \epsilon^{n} \Lambda_{i \alpha}^{n}
$$

where the notation $\Lambda_{i \alpha}^{3}=\Lambda_{i \beta} \Lambda_{\beta \gamma} \Lambda_{\gamma \alpha}$ and $\Lambda_{i \alpha}^{0}=\delta_{i \alpha}$ has been used. Equation (7) can therefore be rewritten as

$$
\mathcal{G}_{E S}=\frac{\rho}{m(2 \pi)^{3 / 2}\left(\operatorname{det} T_{\alpha \beta}\right)^{1 / 2}} \exp \left(-\frac{1}{2}\left(\sum_{n=0}^{\infty} \epsilon^{n} \Lambda_{i \alpha}^{n}\right) \theta_{\alpha j}^{-1} c_{i} c_{j}\right) .
$$

The summation in the exponent can be rewritten as a product of terms as

$$
\mathcal{G}_{E S}=\frac{\rho}{m(2 \pi)^{3 / 2}\left(\operatorname{det} T_{\beta \gamma}\right)^{1 / 2}} \prod_{n=0}^{\infty} \exp \left(-\frac{1}{2}\left(\epsilon^{n} \Lambda_{i \alpha}^{n}\right) \theta_{\alpha j}^{-1} c_{i} c_{j}\right)
$$




$$
\begin{aligned}
& =\frac{\rho}{m(2 \pi)^{3 / 2}\left(\operatorname{det} T_{\beta \gamma}\right)^{1 / 2}} \exp \left(-\frac{1}{2} \theta_{i j}^{-1} c_{i} c_{j}\right) \prod_{n=1}^{\infty} \exp \left(-\frac{1}{2}\left(\epsilon^{n} \Lambda_{i \alpha}^{n}\right) \theta_{\alpha j}^{-1} c_{i} c_{j}\right) \\
& =\frac{\rho}{m(2 \pi)^{3 / 2}\left(\operatorname{det} T_{\beta \gamma}\right)^{1 / 2}} \exp \left(-\frac{1}{2} \theta_{i j}^{-1} c_{i} c_{j}\right) \prod_{n=1}^{\infty}\left(\sum_{k=0}^{\infty} \frac{\left(-\frac{1}{2} \epsilon^{n} \Lambda_{i \alpha}^{n} \theta_{\alpha j}^{-1} c_{i} c_{j}\right)^{k}}{k !}\right) \\
& =\frac{\rho}{m(2 \pi)^{3 / 2}\left(\operatorname{det} T_{\beta \gamma}\right)^{1 / 2}} \exp \left(-\frac{1}{2} \theta_{i j}^{-1} c_{i} c_{j}\right)\left[1+\left(-\frac{\epsilon}{2} \Lambda_{i \alpha} \theta_{\alpha j}^{-1} c_{i} c_{j}\right)+\mathcal{O}\left(\epsilon^{2}\right)\right] .
\end{aligned}
$$

At this point, using the small scaling parameter, $\epsilon$, the scaled distribution function is assumed to have the form

$$
\mathcal{F}=\mathcal{G}\left(g^{(0)}+\epsilon g^{(1)}+\epsilon^{2} g^{(2)}+\epsilon^{3} g^{(3)}+\cdots\right)
$$

This is then substituted into the scaled kinetic equation using Equation 28), which yields

$$
\frac{\partial \mathcal{F}}{\partial t}+v_{k} \frac{\partial \mathcal{F}}{\partial x_{k}}+=-\frac{\operatorname{Pr}}{\epsilon \tau}\left\{\mathcal{F}-\mathcal{G}\left[1+\left(-\frac{\epsilon}{2} \Lambda_{i \alpha} \theta_{\alpha j}^{-1} c_{i} c_{j}\right)\right]\right\}
$$

where the smallness parameter multiplying the relaxation time is akin to assuming that the collision operator will force general distribution functions towards a Gaussian on a very fast time scale. Equation (30) can be rewritten by gathering terms of similar order in $\epsilon$,

$$
\begin{gathered}
\frac{\operatorname{Pr}}{\tau} \mathcal{G}\left[g^{(0)}-1\right]+\epsilon\left[\frac{\partial g^{(0)} \mathcal{G}}{\partial t}+v_{k} \frac{\partial g^{(0)} \mathcal{G}}{\partial x_{k}}-\frac{\operatorname{Pr}}{\tau}\left\{\left(\frac{1}{2} \mathcal{G} \Lambda_{i \alpha} \theta_{\alpha j}^{-1} c_{i} c_{j}\right)-g^{(1)} \mathcal{G}\right\}\right] \\
+\epsilon^{2}[\cdots]+\epsilon^{3}[\cdots]=0
\end{gathered}
$$

It is clear that the zeroth-order solution to this equation is $g^{(0)}=0$. Retaining first-order terms, the relation

$$
g^{(1)} \mathcal{G}=-\frac{\tau}{\operatorname{Pr}}\left[\frac{\partial \mathcal{G}}{\partial t}+v_{k} \frac{\partial \mathcal{G}}{\partial x_{k}}-\frac{\operatorname{Pr}}{2 \tau} \mathcal{G} \Lambda_{i \alpha} \theta_{\alpha j}^{-1} c_{i} c_{j}\right]
$$

is obtained. Substitution of this relation into the unscaled kinetic equation (i.e., using $\mathcal{F}=\mathcal{G}\left(1+g^{(1)}\right)$ ) and taking moments using the weighting functions $\mathbf{M}=\left[m, m v_{i}, m v_{i} v_{j}\right]$ leads to

$$
\left\langle\mathbf{M} \frac{\partial \mathcal{G}}{\partial t}\right\rangle+\left\langle\mathbf{M} \frac{\partial\left(g^{(1)} \mathcal{G}\right)}{\partial t}\right\rangle+\left\langle\mathbf{M} v_{k} \frac{\partial \mathcal{G}}{\partial x_{k}}\right\rangle+\left\langle\mathbf{M} v_{k} \frac{\partial\left(g^{(1)} \mathcal{G}\right)}{\partial x_{k}}\right\rangle=\frac{\operatorname{Pr}}{\tau}\left\langle\mathbf{M}\left(G_{E S}-G\right)\right\rangle-\frac{\operatorname{Pr}}{\tau}\left\langle\mathbf{M}\left(g^{(1)} \mathcal{G}\right)\right\rangle .
$$

The second term on the left-hand side, as well as the second term on the right-hand side, must be equal to zero. This is because solution consistency dictates that $\left\langle\mathbf{M}\left(g^{(1)} \mathcal{G}\right)\right\rangle$ cannot alter the zeroth-order values of $\langle\mathbf{M G}\rangle$. Once the moments have been computed, Equation $(33)$ can be written as

$$
\frac{\partial \mathbf{U}}{\partial t}+\frac{\partial \overline{\mathbf{F}}(\mathbf{U})}{\partial x_{k}}+\frac{\partial}{\partial x_{k}}\left\langle\mathbf{M} v_{k} g^{(1)} \mathcal{G}\right\rangle=\frac{\mathbf{C}}{\tau}\left(\mathbf{U}_{E S}-\mathbf{U}\right)
$$

where $\mathbf{U}$ is the solution state, $\mathbf{U}_{E S}$ is the solution state variables obtained from the ellipsoidal statistical distribution function, $\overline{\mathbf{F}}$ is the flux diad and $\mathbf{C}$ is a vector arising from the collision operator. It is therefore clear that $\left\langle\mathbf{M} v_{k} g^{(1)} \mathcal{G}\right\rangle$ must be determined. The same consistency argument made earlier shows that the moments $\left\langle m v_{i} g^{(1)} \mathcal{G}\right\rangle$ and $\left\langle m v_{i} v_{j} g^{(1)} \mathcal{G}\right\rangle$ must be zero and that $\left\langle m v_{i} v_{j} v_{k} g^{(1)} \mathcal{G}\right\rangle=\left\langle m c_{i} c_{j} c_{k} g^{(1)} \mathcal{G}\right\rangle$. It is this moment which must be determined. This can be written as

$$
\left\langle m c_{i} c_{j} c_{k} g^{(1)} \mathcal{G}\right\rangle=-\frac{\tau}{\operatorname{Pr}}\left\langle m c_{i} c_{j} c_{k}\left(\frac{\partial \mathcal{G}}{\partial t}+v_{l} \frac{\partial \mathcal{G}}{\partial x_{l}}-\frac{\operatorname{Pr}}{2 \tau} \mathcal{G} \Lambda_{l \alpha} \theta_{\alpha m}^{-1} c_{l} c_{m}\right)\right\rangle .
$$

The moment $\left\langle m c_{i} c_{j} c_{k} c_{l} c_{m} \frac{\operatorname{Pr}}{2 \tau} \mathcal{G} \Lambda_{l \alpha} \theta_{\alpha m}^{-1}\right\rangle$ represents the integral of odd functions and is thus equal to zero. The completion of this derivation, therefore, requires the calculation of $\left\langle m c_{i} c_{j} c_{k} \frac{\partial \mathcal{G}}{\partial t}\right\rangle$ and $\left\langle m c_{i} c_{j} c_{k} v_{l} \frac{\partial \mathcal{G}}{\partial x_{l}}\right\rangle$. These integrations will be demonstrated separately, beginning with $\left\langle m c_{i} c_{j} c_{k} \frac{\partial \mathcal{G}}{\partial t}\right\rangle$. This integration will make use of the fact that

$$
\frac{1}{\mathcal{G}} \frac{\partial \mathcal{G}}{\partial t}=\frac{1}{\mathcal{G}} \frac{\partial \mathcal{G}}{\partial \rho} \frac{\partial \rho}{\partial t}+\frac{1}{\mathcal{G}} \frac{\partial \mathcal{G}}{\partial u_{i}} \frac{\partial u_{i}}{\partial t}+\frac{1}{\mathcal{G}} \frac{\partial \mathcal{G}}{\partial \Theta_{i j}} \frac{\partial \Theta_{i j}}{\partial t} .
$$


The derivatives of the Gaussian distribution function with respect to the quantities $\rho, u_{i}$ and $\theta_{i j}$ can be shown to be

$$
\begin{aligned}
\frac{1}{G} \frac{\partial \mathcal{G}}{\partial \rho} & =\frac{1}{\rho} \\
\frac{1}{G} \frac{\partial \mathcal{G}}{\partial u_{i}} & =\Theta_{i k}^{-1} c_{k} \\
\frac{1}{G} \frac{\partial \mathcal{G}}{\partial \Theta_{i j}} & =-\frac{1}{2} \frac{1}{\operatorname{det} \Theta_{\gamma \delta}} \frac{\partial \operatorname{det} \Theta_{\gamma \delta}}{\partial \Theta_{i j}}-\frac{1}{2} \frac{\partial \Theta_{k l}^{-1}}{\partial \Theta_{i j}} c_{k} c_{l} .
\end{aligned}
$$

This results in the following expression

$$
\begin{aligned}
\left\langle m c_{i} c_{j} c_{k} \frac{\partial \mathcal{G}}{\partial t}\right\rangle= & \overbrace{\left\langle m c_{i} c_{j} c_{k} \frac{1}{\rho} \mathcal{G} \frac{\partial \rho}{\partial t}\right\rangle}^{\text {odd }}+\left\langle m c_{i} c_{j} c_{k} \Theta_{\alpha l}^{-1} c_{l} \mathcal{G} \frac{\partial u_{\alpha}}{\partial t}\right\rangle \\
& +\left\langle m c_{i} c_{j} c_{k}\left[-\frac{1}{2} \frac{1}{\operatorname{det} \Theta_{\gamma \delta}} \frac{\partial \operatorname{det} \Theta_{\gamma \delta}}{\partial \Theta_{\alpha \beta}}-\frac{1}{2} \frac{\partial \Theta_{\gamma \delta}^{-1}}{\partial \Theta_{\alpha \beta}} c_{\gamma} c_{\delta}\right] \mathcal{G} \frac{\partial \Theta_{\alpha \beta}}{\partial t}\right\rangle \\
\text { odd } & \Theta_{\alpha l}^{-1}\left\langle c_{i} c_{j} c_{k} c_{l} \mathcal{G}\right\rangle \frac{\partial u_{\alpha}}{\partial t} \\
= & \Theta_{\alpha l}^{-1} \frac{\left[P_{i j} P_{k l}+P_{i k} P_{j l}+P_{i l} P_{j k}\right]}{\rho}\left\{-u_{\beta} \frac{\partial u_{\alpha}}{\partial x_{\beta}}-\frac{1}{\rho} \frac{\partial P_{\alpha \beta}}{\partial x_{\beta}}\right\} \\
= & -\frac{1}{\rho}\left[P_{i j} \frac{\partial P_{k \beta}}{\partial x_{\beta}}+P_{i k} \frac{\partial P_{j \beta}}{\partial x_{\beta}}+P_{j k} \frac{\partial P_{i \beta}}{\partial x_{\beta}}\right] \\
& -\left[P_{i j} u_{\beta} \frac{\partial u_{k}}{\partial x_{\beta}}+P_{i k} u_{\beta} \frac{\partial u_{j}}{\partial x_{\beta}}+P_{j k} u_{\beta} \frac{\partial u_{i}}{\partial x_{\beta}}\right] .
\end{aligned}
$$

The moment $\left\langle m c_{i} c_{j} c_{k} v_{l} \frac{\partial \mathcal{G}}{\partial x_{l}}\right\rangle$ can be integrated as

$$
\begin{aligned}
\left\langle m c_{i} c_{j} c_{k} v_{l} \frac{\partial \mathcal{G}}{\partial x_{l}}\right\rangle= & \frac{\partial}{\partial x_{l}}\left\langle m c_{i} c_{j} c_{k} v_{l} \mathcal{G}\right\rangle-\left\langle\mathcal{G} \frac{\partial}{\partial x_{l}}\left[m c_{i} c_{j} c_{k} v_{l}\right]\right\rangle \\
= & \frac{\partial}{\partial x_{l}}[\left\langle m c_{i} c_{j} c_{k} c_{l} \mathcal{G}\right\rangle+u_{l} \underbrace{\left\langle m c_{i} c_{j} c_{k} \mathcal{G}\right\rangle}_{\text {odd }}] \\
& -\left\langle\mathcal{G}\left[m c_{i} c_{j} v_{l} \frac{\partial c_{k}}{\partial x_{l}}+m c_{i} c_{k} v_{l} \frac{\partial c_{j}}{\partial x_{l}}+m c_{j} c_{k} v_{l} \frac{\partial c_{i}}{\partial x_{l}}\right]\right\rangle \\
= & \frac{\partial}{\partial x_{l}}\left\langle m c_{i} c_{j} c_{k} c_{l} \mathcal{G}\right\rangle+\left\langle\mathcal{G}\left[m c_{i} c_{j} v_{l} \frac{\partial u_{k}}{\partial x_{l}}+m c_{i} c_{k} v_{l} \frac{\partial u_{j}}{\partial x_{l}}+m c_{j} c_{k} v_{l} \frac{\partial u_{i}}{\partial x_{l}}\right]\right\rangle \\
= & \frac{\partial}{\partial x_{l}}\left\langle m c_{i} c_{j} c_{k} c_{l} \mathcal{G}\right\rangle+\left\langle\mathcal{G}\left[m c_{i} c_{j} c_{l} \frac{\partial u_{k}}{\partial x_{l}}+m c_{i} c_{k} c_{l} \frac{\partial u_{j}}{\partial x_{l}}+m c_{j} c_{k} c_{l} \frac{\partial u_{i}}{\partial x_{l}}\right]\right\rangle \\
& +u_{l}\left\langle\mathcal{G}\left[m c_{i} c_{j} \frac{\partial u_{k}}{\partial x_{l}}+m c_{i} c_{k} \frac{\partial u_{j}}{\partial x_{l}}+m c_{j} c_{k} \frac{\partial u_{i}}{\partial x_{l}}\right]\right\rangle \\
= & \frac{\partial}{\partial x_{l}}\left[\frac{P_{i j} P_{k l}+P_{i k} P_{j l}+P_{i l} P_{j k}}{\rho}\right]+u_{l}\left[P_{i j} \frac{\partial u_{k}}{\partial x_{l}}+P_{i k} \frac{\partial u_{j}}{\partial x_{l}}+P_{j k} \frac{\partial u_{i}}{\partial x_{l}}\right] .
\end{aligned}
$$

This series of steps made use of the facts that $\frac{\partial v_{i}}{\partial x_{j}}=0$ and $\frac{\partial c_{k}}{\partial x_{l}}=-\frac{\partial u_{k}}{\partial x_{l}}$.

Combination of equations 40 and 41 , followed by some simple algebra, leads to the relation

$$
Q_{i j k}=\left\langle m c_{i} c_{j} c_{k} g^{(1)} \mathcal{G}\right\rangle=-\frac{\tau}{\operatorname{Pr}}\left[P_{k l} \frac{\partial}{\partial x_{l}}\left(\frac{P_{i j}}{\rho}\right)+P_{j l} \frac{\partial}{\partial x_{l}}\left(\frac{P_{i k}}{\rho}\right)+P_{i l} \frac{\partial}{\partial x_{l}}\left(\frac{P_{j k}}{\rho}\right)\right],
$$

$$
8 \text { of } 18
$$


which is exactly the same result as obtained by the previous derivation based on a perturbative expansion applied directly to the moment equations. These same thermal diffusion terms can also easily be used with the diatomic form of the Gaussian moment equations.

\section{III.C. Solid-Wall Boundary Conditions}

Appropriate solid-wall boundary conditions for the Gaussian closure are not immediately obvious. One technique for determining the solution at a wall is to assume that there exists a Knudsen layer next to the solid surface. ${ }^{6}$ In this infinitesimally thin layer, the fluid exists as a combination of the distribution function defining incoming particles from the interior flow field and a distribution function defining reflected particles arising from the wall. For example, for a solid wall extending in the x-direction with a fluid above it, all the particles with negative $y$-direction velocities in the Knudsen layer will come from the neighbouring fluid with statistical properties defined by the Gaussian. In order to model the particle interaction with the wall, an accommodation coefficient, $0 \leq \alpha \leq 1$, is then defined. If $\alpha$ is zero (specular reflection), the incoming particles will simply be reflected specularly from the wall back into the Knudsen layer. For $\alpha=1$ (diffuse reflection), incoming particles are fully accommodated and will therefore come into thermodynamic equilibrium with the wall before being released from the wall and will re-enter the Knudsen layer with the statistical properties of a Maxwell-Boltzmann distribution defined by a wall temperature, $T_{w}$. For any intermediate $\alpha$ value, the reflected particles will enter the Knudsen layer as a combination of the two cases. The resulting distributions function for the Knudsen layer is then given by

$$
\mathcal{F}_{\mathrm{Kn}}=\mathcal{F}^{+}+\mathcal{F}^{-},
$$

where $\mathcal{F}^{+}$and $\mathcal{F}^{-}$are given by

$$
\begin{gathered}
\mathcal{F}^{-}=\left\{\begin{array}{cl}
\mathcal{G}_{b}\left(v_{x}, v_{y}, v_{z}\right) & \text { for } v_{y}<0, \\
0 & \text { for } v_{y}>0,
\end{array}\right. \\
\mathcal{F}^{+}=\left\{\begin{array}{cl}
\alpha \mathcal{M}_{w}\left(v_{x}, v_{y}, v_{z}\right)+(1-\alpha) \mathcal{G}_{b}\left(v_{x},-v_{y}, v_{z}\right) & \text { for } v_{y}>0, \\
0 & \text { for } v_{y}<0,
\end{array}\right.
\end{gathered}
$$

and where $\mathcal{G}_{b}$ is Gaussian distribution at the edge of the Knudsen layer and $\mathcal{M}_{w}$ is the Maxwellian defining particles which are fully accommodated by the wall. By assuming that the bulk y-direction velocity of the fluid immediately above the wall is zero and by imposing the constraint that the net particle flux through the wall must be zero, it is possible to show that the reflected Maxwellian has the form:

$$
\mathcal{M}_{w}\left(v_{x}, v_{y}, v_{z}\right)=\sqrt{\frac{n P_{y y}}{k T_{w}}}\left(\frac{m}{2 \pi k T_{w}}\right)^{\frac{3}{2}} e^{-\left(\frac{m}{2 k T_{w}}\right)\left(\left(v_{x}-\left(u_{x}\right)_{w}\right)^{2}+v y^{2}+v z^{2}\right)},
$$

where $k$ is Boltzmann's constant and $n$ is the number density. All the properties are those of the fluid outside the Knudsen layer with the exception of $T_{w}$ which is the temperature of the wall and $\left(u_{x}\right)_{w}$ which is the $\mathrm{x}$-direction velocity of the wall.

Once this is determined, all of the required moments of $\mathcal{F}_{\mathrm{Kn}}$ for two-dimensional flows can be shown to be

$$
\begin{aligned}
(\rho)_{\mathrm{Kn}}= & \rho\left(1+\frac{\alpha}{2}\left[\sqrt{\frac{m P_{y y}}{\rho k T_{w}}}\right]\right) \\
\left(\rho u_{x}\right)_{\mathrm{Kn}}= & (2-\alpha)\left(\frac{\rho u_{x}}{2}-\frac{\rho P_{x y}}{\sqrt{2 \pi \rho P_{y y}}}\right)+\frac{\alpha}{2} \sqrt{\frac{P_{y y}}{n k T_{w}}}\left(u_{x}\right)_{w} \\
\left(\rho u_{y}\right)_{\mathrm{Kn}}= & 0 \\
\left(P_{x x}\right)_{\mathrm{Kn}}= & (2-\alpha)\left(\frac{P_{x x}}{2}+\frac{\rho\left(u_{x}-\left(u_{x}\right)_{\mathrm{Kn}}\right)^{2}}{2}-P_{x y} \sqrt{\frac{2 \rho}{\pi P_{y y}}}\left(u_{x}-\left(u_{x}\right)_{\mathrm{Kn}}\right)\right) \\
& +\alpha\left(\frac{n_{w} k T_{w}}{2}+\frac{\rho_{w}\left(\left(u_{x}\right)_{w}-\left(u_{x}\right)_{\mathrm{Kn}}\right)^{2}}{2}\right)
\end{aligned}
$$




$$
\begin{aligned}
& \left(P_{x y}\right)_{\mathrm{Kn}}=\alpha\left(\frac{P_{x y}}{2}-\sqrt{\frac{\rho P_{y y}}{2 \pi}}\left(u_{x}-\left(u_{x}\right)_{\mathrm{Kn}}\right)+\sqrt{\frac{\rho_{w} n_{w} k T_{w}}{2 \pi}}\left(\left(u_{x}\right)_{w}-\left(u_{x}\right)_{\mathrm{Kn}}\right)\right) \\
& \left(P_{y y}\right)_{\mathrm{Kn}}=\frac{2-\alpha}{2} P_{y y}+\frac{\alpha}{2} \sqrt{P_{y y} n_{w} k T_{w}} \\
& \left(P_{z z}\right)_{\mathrm{Kn}}=\frac{2-\alpha}{2} P_{z z}+\frac{\alpha}{2} \sqrt{P_{z z} n_{w} k T_{w}} .
\end{aligned}
$$

The thermal diffusion terms which are added to the standard Gaussian closure when used with Knudsenlayer boundary conditions ${ }^{6}$ do not allow for temperature slip between the gas and solid boundary. However this phenomenon is expected for moderate Knudsen-number flows and must be accounted for. Fortunately temperature-slip boundary conditions have been previously studied. ${ }^{27}$ For the present work, the following expression is used

$$
T=T_{w}+g \lambda \frac{\partial T}{\partial n_{i}}
$$

where $T$ is the temperature of the fluid at the boundary, $T_{w}$ is the temperature of the wall, $n_{i}$ is the unit normal to the wall, and $g$ is the slip distance factor given by the expression

$$
g=\frac{10 \pi}{16 \operatorname{Pr}}\left(\frac{2-a}{a}\right) \frac{\gamma}{\gamma+1} .
$$

Here $a$ is a thermal accommodation coefficient which describes the fractional extent to which molecules which impact a surface and are re-emitted from it have their energy adjusted to that of a stream of particles in thermal equilibrium with the wall; $a=0$ corresponds to reflected particles having no change to their energy and $a=1$ corresponds to particles begin fully thermally accommodated by the wall. These boundary conditions can be combined with Knudsen-layer boundary conditions by assuming that particles are emitted from solid boundaries at the temperature predicted by the slip condition of Eq. (47).

\section{Parallel AMR Finite-Volume Scheme}

A parallel high-order Godunov-type finite-volume scheme with block-based adaptive mesh refinement (AMR) has been developed for the solution of the transport equations of the Gaussian closure with diffusive heat transfer for two-dimensional planar flows on multi-block quadrilateral meshes. The hyperbolic numerical fluxes are determined from the approximate solution of a Riemann problem posed in a direction defined by the normal to the cell faces. The left and right solution states for the Riemann problems are determined via a least-squares piece-wise limited linear solution reconstruction procedure with either the Barth-Jesperson or Venkatakrishnan limiters. ${ }^{28,29}$ This provides a second-order-accurate spatial discretization for smooth solutions. In the present algorithm, both Roe- and HLLE-type approximate Riemann solvers ${ }^{30,31}$ are used to solve the Riemann problem and evaluate the numerical flux. Due to the stiffness of the source terms arising from the collision operator, a point-implicit time integration scheme is used. ${ }^{8}$ Elliptic fluxes arising from thermal diffusion are calculated using a diamond-path reconstruction technique. ${ }^{32,33}$

This finite-volume scheme has been implemented with a parallel block-based AMR scheme previously developed by Sachdev et al. ${ }^{34}$ This technique allows initial body-fitted meshes to be automatically refined in areas of interest which are found using physics-based refinement criteria. The scheme has been designed to make efficient use of large-scale distributed-memory parallel clusters. This solver has previously been applied to many continuum and micron-scale flow problems using the traditional Gaussian moment equations. ${ }^{8,35}$

\section{Numerical Results}

Application of the regularized Gaussian closure discussed above to some standard flow problems is now considered for a range of Knudsen numbers. For the calculation of the mean free path required to determine the Knudsen number, the gas is assumed to be comprised of hard spheres ${ }^{3}$ for which

$$
\lambda=\frac{16 \mu}{5(2 \pi \rho p)^{\frac{1}{2}}} .
$$




\section{V.A. Shock-Structure Calculations}

As a preliminary study into the behaviour of the Gaussian closure with the present correction for thermal diffusion, one-dimensional planar shock structures were investigated for shock waves of differing strengths traveling through argon. The internal structure of shocks is characterized by highly non-equilibrium features and represents a significant challenge for any non-equilibrium model. The results of the present investigation can be seen in Figures 1 and 2 where normalized density and one entry on the generalized heat-flux tensor, $Q_{x x x}$, are plotted. The density is normalized by the relation

$$
\rho^{\star}=\frac{\rho-\rho_{u}}{\rho_{d}-\rho_{u}},
$$

where $\rho_{u}$ and $\rho_{d}$ are the up- and down-stream densities respectively. The heat-flux entry $Q_{x x x}$ is normalized as

$$
Q_{x x x}^{\star}=\frac{Q_{x x x}}{\left(p_{x x} \sqrt{\frac{p_{x x}}{\rho}}\right)} .
$$

Results for shock Mach numbers, $\mathrm{M}_{\mathrm{s}}$, of $1.2,1.5$, and 2 are shown.

Unlike the Euler equations, the standard Gaussian moment equations do not predict discontinuous shock structures for all Mach numbers. Rather, for Mach numbers lower than a critical value, a smooth transition between up- and down-stream states is predicted; this can be observed in Figure 1(a). However, due to hyperbolicity of the equations, above a critical Mach number a discontinuity will appear. This discontinuity can clearly be seen in the $\mathrm{M}_{\mathrm{s}}=1.5$ and $\mathrm{M}_{\mathrm{s}}=2$ cases of Figures $1(\mathrm{~b})$ and $1(\mathrm{c})$.

Conversely, it can be seen that the proposed correction for thermal diffusion leads to smooth (fully dispersed) shock transitions for a wide range of shock Mach numbers. It can also be seen that for lower-Machnumber cases, the agreement between the regularized Gaussian moment equations and DSMC calculations is generally quite good. However, for the $\mathrm{M}_{\mathrm{s}}=2$ case, the generalized Gaussian and the DSMC results begin to differ. A comparison of the predicted normalized values of $Q_{x x x}$ for the regularized Gaussian closure and DSMC are shown in Figure2 2 Again it can be seen that there is good agreement, especially for shock Mach numbers of 1.2 and 1.5 .

\section{V.B. Heat Transfer Between Infinite Plates}

A good test case to verify the proper implementation of the temperature-slip boundary conditions is heat transfer between infinite isothermal plates. This case can be used both to ensure that the correct temperature slip is predicted at the boundary and to study the predicted heat transfer between the plates over a range of flow regimes and Knudsen numbers. It is expected that for low-Knudsen-number situations, the temperature slip between the boundary and the fluid should be imperceptible. It is only as the Knudsen number increases into the slip-flow regime that any appreciable slip should be observed. Finally, in the limit of infinite Knudsen number, the temperature slip should be perfect and the fluid should have a uniform temperature throughout.

Figure 3(a) shows the temperature of argon gas at one of the solid plates for the situation where the plate temperatures differ by $20 \mathrm{~K}$. This temperature is normalized by the formula

$$
T^{\star}=\frac{T-T_{m}}{T_{w}-T_{m}},
$$

where $T^{\star}$ is the normalized temperature, $T$ is the temperature of the gas at the wall, $T_{w}$ is the temperature of the solid wall, and $T_{m}$ is the temperature of the gas midway between the plates. It can be seen that all of these expected behaviours detailed above are observed; there is no noticeable slip $\left(T^{\star} \approx 1\right)$ in the lowKnudsen-number situations and the slip approaches perfect ( $T^{\star}$ approaches zero) as the Knudsen number increases.

Non-continuum effects are also very evident when the predicted heat transfer between the plates is considered. This data has been plotted in Figure 3(b). This figure shows the predicted heat flux for the continuum Navier-Stokes equations, the Navier-Stokes equations with slip-temperature boundary conditions, solutions obtained using the regularized Gaussian moment equations with slip boundary conditions, and the heat flux predicted by integration of two half Maxwellians emitted from the solid walls (the free-molecular solution); these have all been normalized with respect to the free-molecular solution. It can be seen that the extended Gaussian moment equations together with slip-temperature boundary condition transition from the 


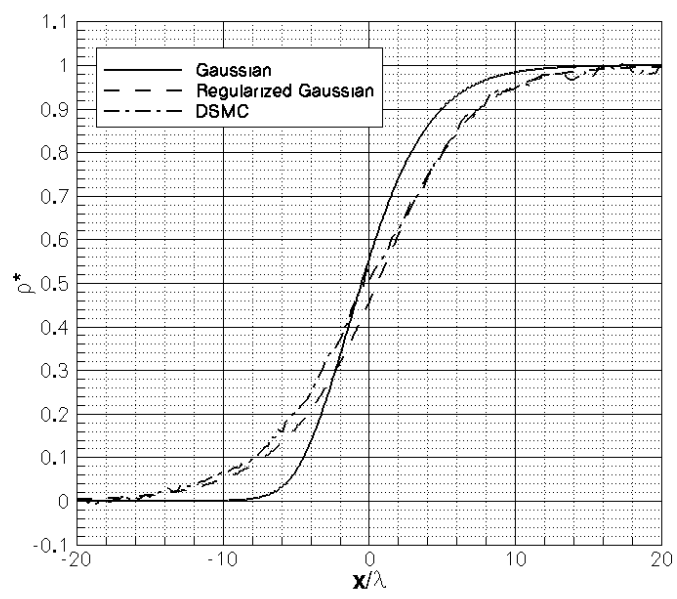

(a)

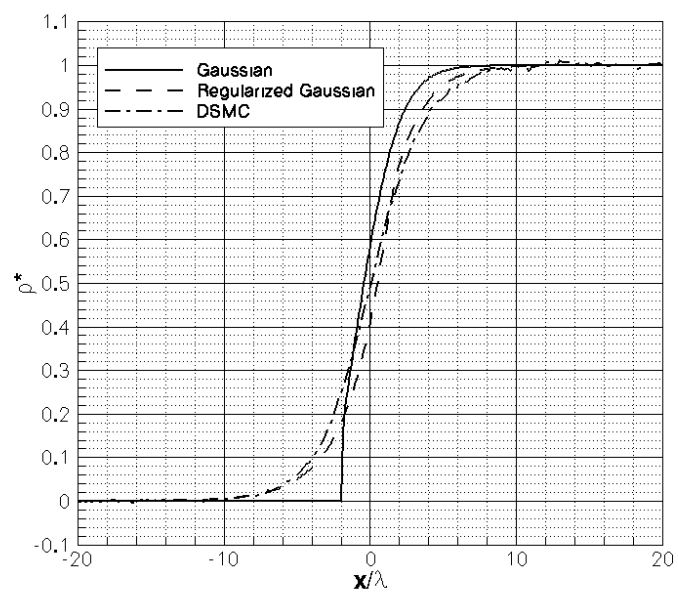

(b)

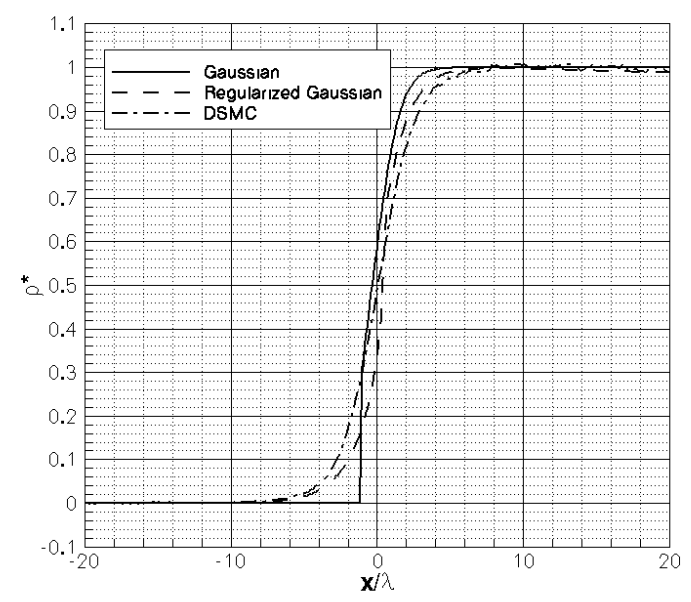

(c)

Figure 1. Normalized density variations through shock waves with shock Mach numbers of (a) $M_{\mathrm{S}}=1.2$, (b) $M_{\mathrm{S}}=1.5$, and (c) $M_{\mathrm{S}}=2$.

continuum solution to a free-molecular limit which is slightly higher than the true free-molecular solution. This is a consequence of the derivation used to calculate the slip distance (Eq. 48). It is possible to "tune" the slip distance so as to obtain the correct free-molecular heat flux for the case of heat transfer between two plates, however it would only be correct in this situation. It seems more advisable to use the equation for slip distance given above as this is derived from more physical arguments.

\section{V.C. Subsonic Flow Past a Circular Cylinder}

In previous studies by the authors, ${ }^{8,35}$ low-speed flow past a circular cylinder as predicted by the standard Gaussian moment equations has been considered. There is a reasonable amount of data and theory available in the literature regarding this situation. In particular the coefficient of drag, $C_{d}$, is available for continuum, transitional, and free-molecular flow regimes. In the previous studies comparison between the Gaussian equations, experimental results collected by Coudeville et al. ${ }^{36}$ and an approximate solution developed by Patterson $^{37}$ for airflow at a speed ratio of 0.027 and 0.107 have shown good agreement for low-Knudsen- 


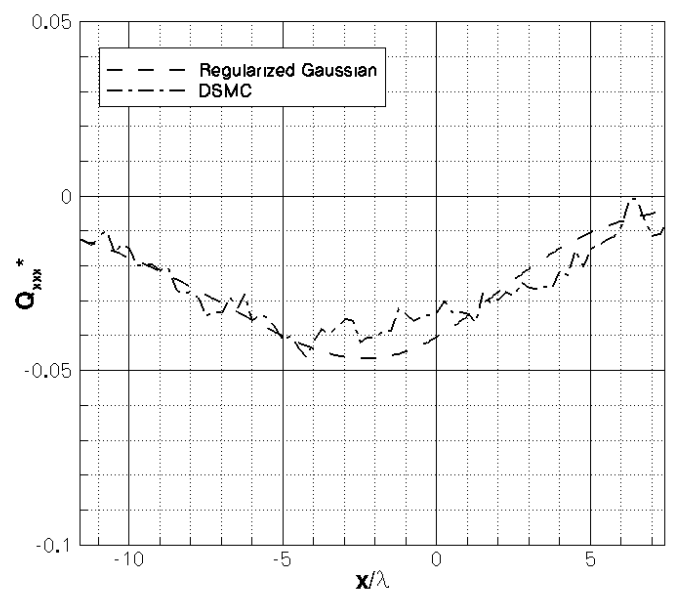

(a)

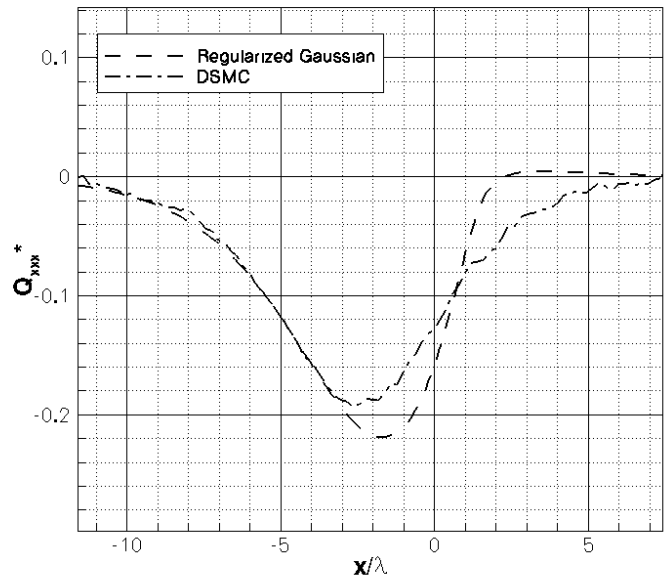

(b)

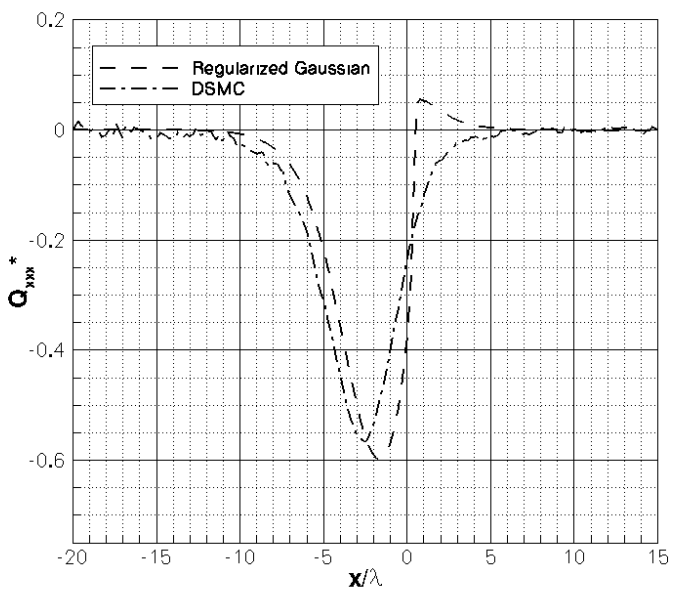

(c)

Figure 2. Normalized heat-flux variations through shock waves with shock Mach numbers of (a) $M_{\mathrm{S}}=1.2$, (b) $M_{\mathrm{S}}=1.5$, and (c) $M_{\mathrm{S}}=2$.

number situations ${ }^{c}$ However, as the Knudsen increases, the Gaussian moment equations seem to over-predict the coefficient of drag. Figure 4 shows the results of this previous study.

With hopes of obtaining better agreement for higher-Knudsen-number situations, a portion of these flow situations were re-computed using the regularized Gaussian moment equations. Figure 5 shows the results of this effort.

It was found that the slip-temperature boundary conditions described above introduce numerical difficulties for the cylinder flows and often result in aphysical negative temperatures at the boundary when a large temperature gradient is present. In order to obtain solutions, it was found that isothermal boundary conditions had to be imposed at the cylinder wall. This was carried out by maitaining a fixed or constant value for the wall temperature, $T_{w}$, in Eq. (46). In Figure 5, it can be seen that the addition of thermal diffusion seems to reduce the predicted coefficient of drag for this case. Unfortunately, in the transition regime, the regularized Gaussian equations now seem to under-predict the coefficient of drag. This could be due to the use of the isothermal boundary conditions at the cylinder wall. It was shown in the previous

${ }^{\mathrm{c}}$ The speed ratio is the ratio of the bulk speed to the most probable random speed of a particle, it differs from the Mach number by a constant of order unity. 


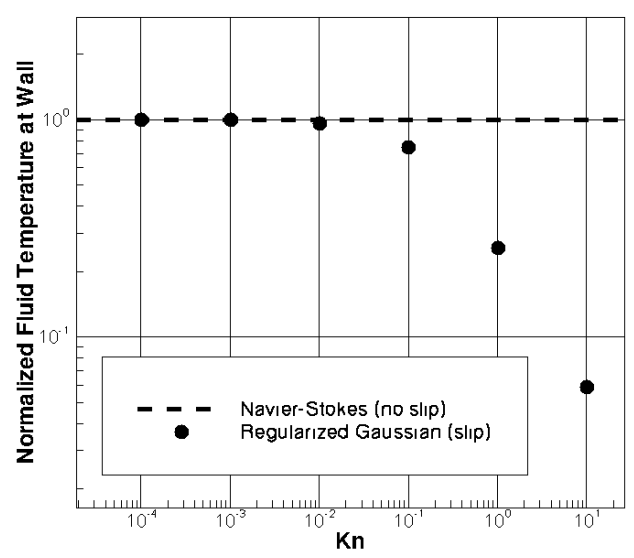

(a)

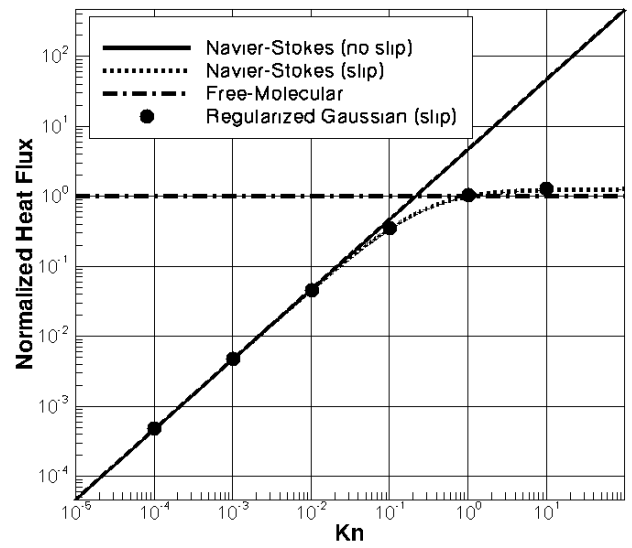

(b)

Figure 3. Heat transfer between infinite plates: (a) normalized temperature of the gas the wall, (b) predicted heat flux between the plates.

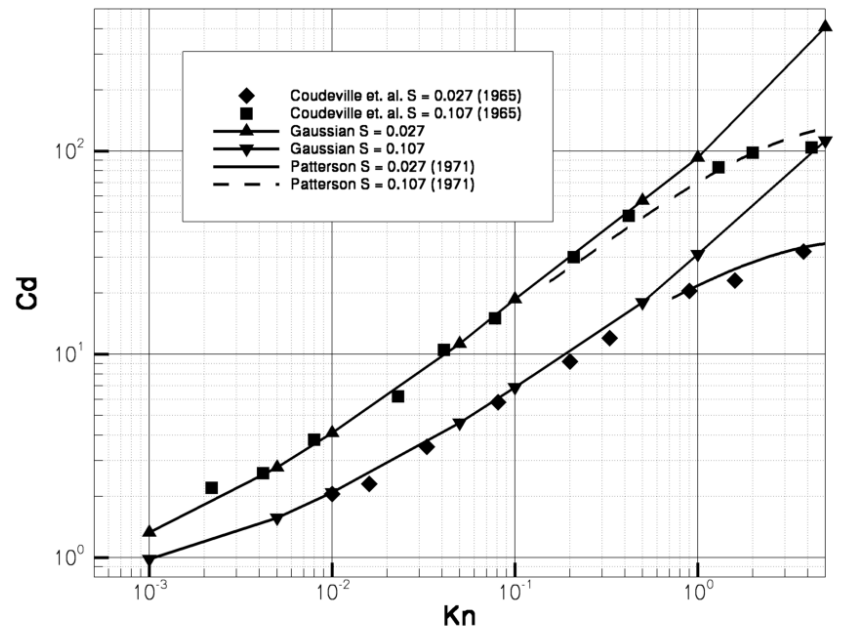

Figure 4. Coefficients of drag for airflow past a circular cylinder computed using the standard Gaussian moment closure on a body-fitted mesh as compared to experimental results of Coudeville et al. ${ }^{36}$ and Patterson's ${ }^{37}$ approximate analytic expression.

infinite-plate calculations that slip-boundary conditions reduce the heat transfer from a boundary in the slipand free-molecular-flow regimes. Inclusion of more realistic boundary conditions may improve the results in this regime.

The magnitude of the differences caused by thermal diffusion may seem surprising given that the lowspeed nature of the flow does not produce a large variation in the gas temperature. However, the decreasing spatial scales associated with higher-Knudsen-number situations leads to large gradients, even for small total differences in temperature.

\section{V.D. Transonic Flow Past a NACA0012 Micro-Airfoil}

Lastly, the application of the Gaussian closure to the prediction of transonic steady flow around a NACA0012 micro-airfoil at zero angle of attack is now considered. For the case presented, the free-stream values of the 


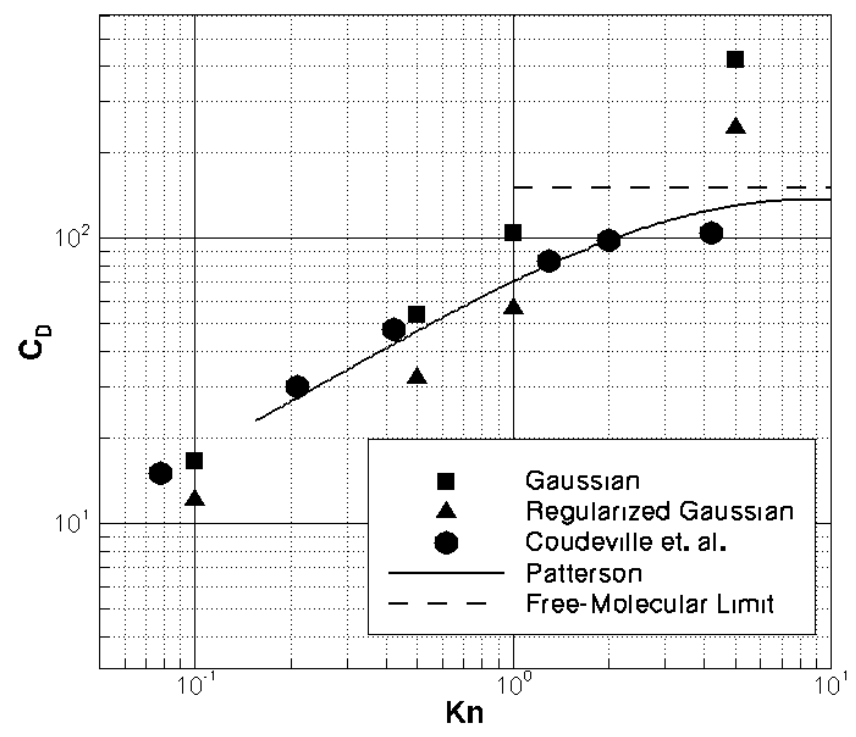

Figure 5. Coefficients of drag for $S=0.027$ airflow past a circular cylinder computed using the standard and regularized Gaussian moment closure on a body-fitted mesh as compared to experimental results of Coudeville et al. ${ }^{36}$ and Patterson's ${ }^{37}$ approximate analytic expression.

flow Mach number, temperature, and density are $0.8,257 \mathrm{~K}$, and $1.161 \times 10^{-4} \mathrm{Kg} / \mathrm{m}^{3}$, respectively, and the chord length of the airfoil is $0.04 \mathrm{~m}$. These conditions correspond to a Knudsen number of 0.017 are the same as those considered in a study of the standard Gaussian moment equations by Suzuki and van Leer. ${ }^{38}$ In this previous study, Suzuki and van Leer reproduce results for this case obtained using a DSMC-based scheme obtained by Sun and Boyd ${ }^{5}$ and experimental results obtained by Allegre, Raffin and Lengrand. ${ }^{39}$ Both of these results are again reproduced here in Figures 6(c) and 6(d). For comparison, Figures 6(a) and 6(b) show results obtained using the standard and regularized Gaussian moment equations respectively. Again, due to problems with negative temperatures at the boundary, slip-temperature boundary conditions could not be used and isothermal Knudsen-layer boundary conditions were used instead.

It is evident from the comparisons Figures $6(\mathrm{c}) 6(\mathrm{~b})$ that the simulations obtained using the standard Gaussian equations agree well with DSMC and experiment at the leading edge, however the density seems to be very under-predicted along the length of the airfoil. A similar finding was reported in the earlier work by Suzuki and van Leer. ${ }^{38}$ However, for this case, the inclusion of thermal diffusion greatly improves the agreement with both DSMC and experiment, particularly towards the trailing edge of the airfoil. In general, the agreement between DSMC and the regularized Gaussian equations seems very good.

\section{Discussion}

It has been shown that the current extension to the standard Gaussian moment equations can lead to improved results for transition-regime flows in which heat transfer has a significant effect. There are, however, several issues in the derivation which should be mentioned. First, the assumption that deviations from the moment quantities predicted by the Gaussian closure will be attenuated rapidly by collisional processes which lead to the introduction of $\epsilon$ on the right-hand side of Eq. 21) is not well founded as relaxation times for higher-order moment tend to be larger than for lower-order moments. ${ }^{11}$

There is a related problem with the derivation using the kinetic equation. Remembering the relation between the Prandtl number and the parameter $\nu$ in the ellipsoidal statistical collision model $((1-\nu) \operatorname{Pr}=1)$, it can be seen that the assumption that $(1-\nu)=\epsilon \ll 1$ is equivalent to assuming that the Prandtl number of the gas is much larger than unity. This is in contrast with most gases.

It should also be mentioned that the ellipsoidal statistical collision model's adherence to Boltzmann's H 


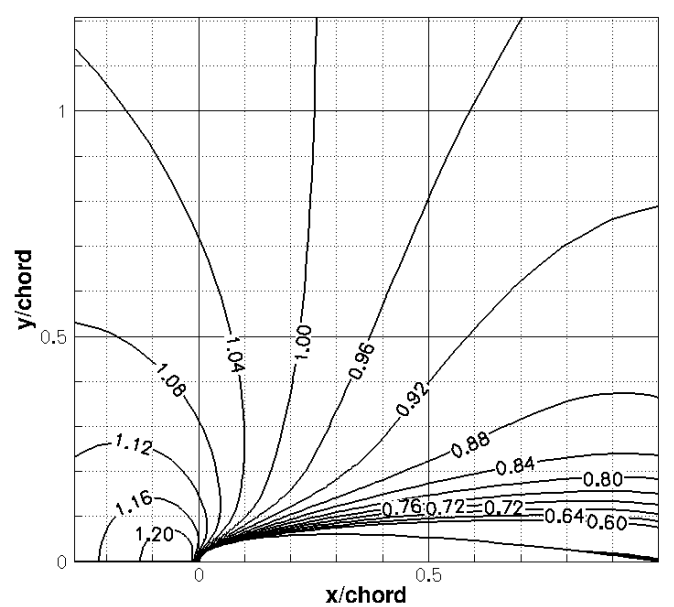

(a)

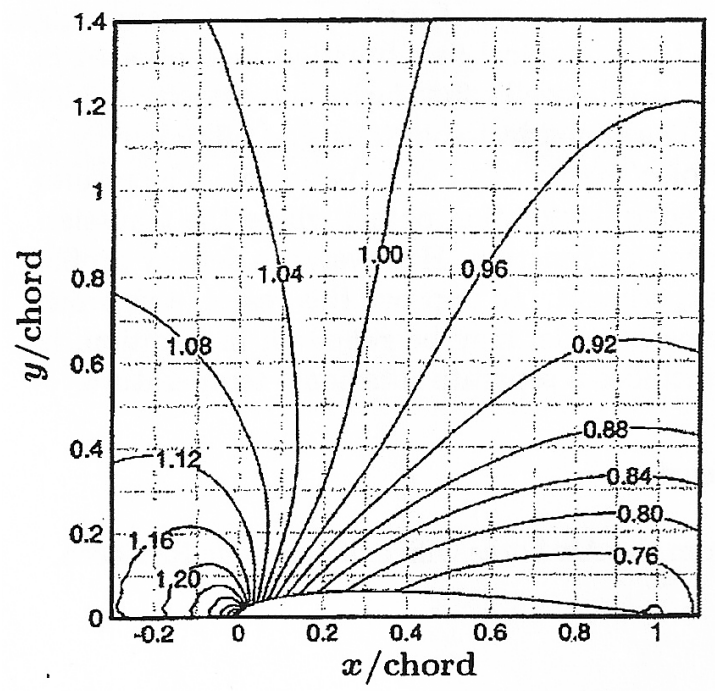

(c)

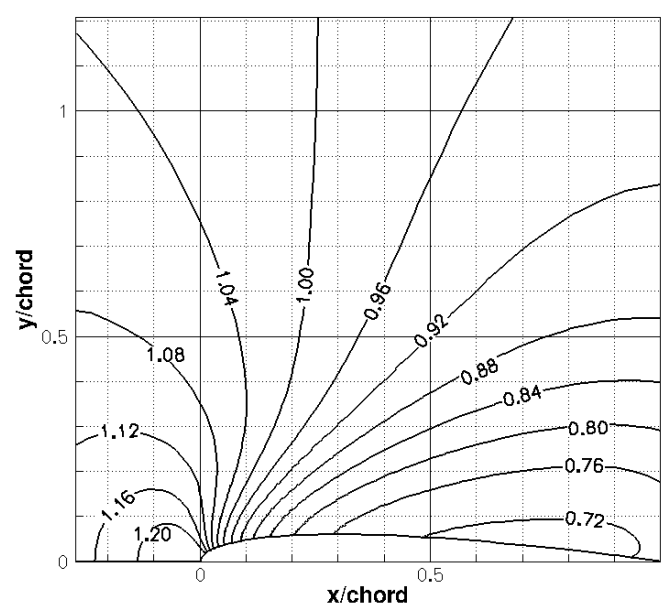

(b)

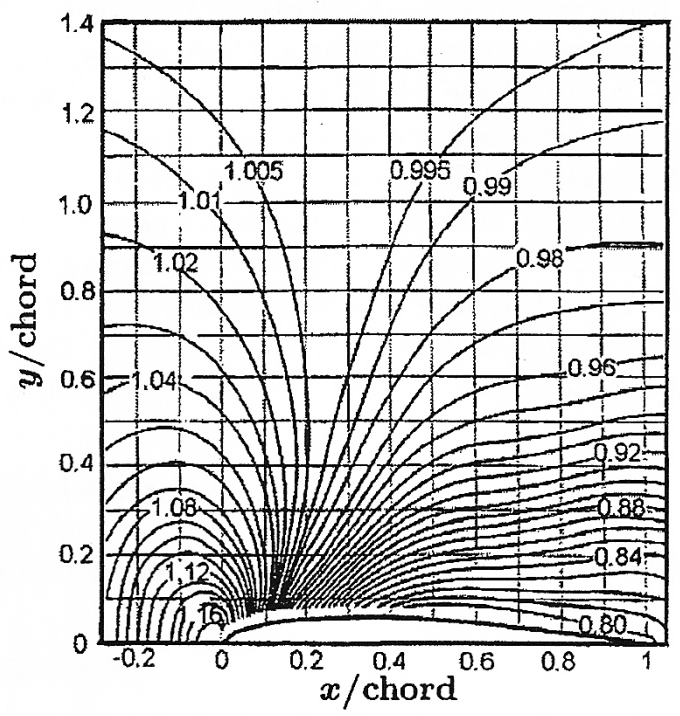

(d)

Figure 6. Comparison of th normalized density contours around a NACA0012 micro-airfoil: (a) predictions of the standard Gaussian moment equations, (b) predictions of the the regularized Gaussian moment equations, (c) predictions of the DSMC-based method of Sun and Boyd ${ }^{5}$ and (d) experimental data of Allegre, Raffin and Lengrand. ${ }^{39}$

theorem has only been demonstrated for monatomic gases. However, experience obtained during this study suggest that for the present purposes it also leads to good results for diatomic gases.

\section{Conclusions}

This study has presented a derivation of an extended fluid-dynamic model for micron-scale flows with heat transfer based on the Gaussian moment closure. The derivation makes use of a Chapman-Enskog expansion about either the moment equations or the kinetic equation. This results in additional elliptic terms describing a generalized non-equilibrium heat-flux tensor. Boundary conditions using a combination of temperature-slip and Knudsen-layer approximations have been presented. A Godunov-type finite-volume scheme has been used to obtain numerical solutions for several flow problems including shock-structure calculations, predictions of heat transfer between parallel plates, flow past a circular cylinder, and flow past a micro-airfoil. The capabilities of the method for describing non-equilibrium flows has been illustrated and 
it has been shown that thermal diffusion can have a significant effect on many transition-regime micron-scale flows. Future work will involve further assessment of the proposed model.

\section{References}

${ }^{1}$ Beskok, A. and Karniadakis, G. E., "Simulation of Heat and Momentum Transfer in Complex Microgeometries," Journal of Thermophysics and Heat Transfer, Vol. 8, No. 4, 1994, pp. 647-655.

${ }^{2}$ Harley, J. C., Huang, Y., Bau, H. B., and Zemel, J. N., "Gas-Flow in Micro-Channels," Journal of Fluid Mechanics, Vol. 284, 1995, pp. 257-274.

${ }^{3}$ Bird, G. A., Molecular Gas Dynamics and the Direct Simulation of Gas Flows, Clarendon Press, Oxford, 1994.

${ }^{4}$ Fan, J. and Shen, C., "Statistical Simulation of Low-Speed Rarefied Gas Flow," Journal of Computational Physics, Vol. 167, 2001, pp. 393-412.

${ }^{5}$ Sun, Q. and Boyd, I. D., "A Direct Simulation Method for Subsonic, Mircoscale Gas Flow," Journal of Computational Physics, Vol. 179, 2002, pp. 400-425.

${ }^{6} \mathrm{Grad}, \mathrm{H}$. , "On the Kinetic Theory of Rarefied Gases," Communications on Pure and Applied Mathematics, Vol. 2, 1949, pp. 331-407.

${ }^{7}$ Levermore, C. D., "Moment Closure Hierarchies for Kinetic Theories," Journal of Statistical Physics, Vol. 83, 1996, pp. 1021-1065.

${ }^{8}$ McDonald, J. and Groth, C. P. T., "Numerical Modeling of Micron-Scale Flows Using the Gaussian Moment Closure," Paper 2005-5035, AIAA, June 2005.

${ }^{9}$ Groth, C. P. T., Gombosi, T. I., Roe, P. L., and Brown, S. L., "Gaussian-Based Moment-Method Closures for the Solution of the Boltzmann Equation," Proceedings of the Fifth International Conference on Hyperbolic Problems - Theory, Numerics, Applications, University of New York at Stony Brook, Stony Brook, New York, U.S.A., June 13-17, 1994, edited by J. Glimm, M. J. Graham, J. W. Grove, and B. J. Plohr, World Scientific, New Jersey, 1996, pp. 339-346.

${ }^{10}$ Groth, C. P. T., Roe, P. L., Gombosi, T. I., and Brown, S. L., "On the Nonstationary Wave Structure of a 35-Moment Closure for Rarefied Gas Dynamics," Paper 95-2312, AIAA, June 1995.

${ }^{11}$ Struchtrup, H. and Torrilhon, M., "Regularization of Grad's 13 Moment Equations: Derivation and Linear Analysis," Physics of Fluids, Vol. 15, 2003, pp. 2668-2680.

${ }^{12}$ Chapman, S. and Cowling, T. G., The Mathematical Theory of Non-Uniform Gases, Cambridge University Press, Cambridge, 1960.

${ }^{13}$ Burgers, J. M., Flow Equations for Composite Gases, Academic Press, New York, 1969.

${ }^{14}$ Gombosi, T. I., Gaskinetic Theory, Cambridge University Press, Cambridge, 1994.

${ }^{15}$ Bhatnagar, P. L., Gross, E. P., and Krook, M., "A Model for Collision Processes in Gases. I. Small Amplitude Processes in Charged and Neutral One-Component Systems," Physical Review, Vol. 94, No. 3, 1954, pp. 511-525.

${ }^{16}$ Holway, L. H., "New Statistical Models for Kinetic Theory: Methods of Construction," Physics of Fluids, Vol. 9, No. 9, 1966, pp. 1658-1673.

${ }^{17}$ Andries, P. and Perthame, B., "The ES-BGK Model Equation with Correct Prandtl Number," Rarefied Gas Dynamics, edited by T. J. Bartel and M. A. Gallis, Vol. I, American Institute of Physics, New York, 2001, pp. 30-36.

${ }^{18}$ Maxwell, J. C., "On the Dynamical Theory of Gases," Philosophical Transactions of the Royal Society of London, Vol. 157, 1867, pp. 49-88.

${ }^{19}$ Hertweck, F., "Allgemeine 13-Momenten-Näherung zur Fokker-Planck-Gleichung eines Plasmas," Zeitschrift für Naturforschung, Vol. 20a, 1965, pp. 1243-1255.

${ }^{20}$ Oraevskii, V., Chodura, R., and Feneberg, W., "Hydrodynamic Equations for Plasmas in Strong Magnetic Fields - I Collisionless Approximation," Plasma Physics, Vol. 10, 1968, pp. 819-828.

${ }^{21}$ Holway, L. H., Approximation Procedures for Kinetic Theory, Ph.D. thesis, Harvard University, 1963.

${ }^{22}$ Holway, L. H., "Kinetic Theory of Shock Structure Using an Ellipsoidal Distribution Function," Rarefied Gas Dynamics, edited by J. H. de Leeuw, Vol. I, Academic Press, New York, 1966, pp. 193-215.

${ }^{23}$ Holway, L. H., "The Effect of Collisional Models upon Shock Wave Structure," Rarefied Gas Dynamics, edited by C. L. Brundin, Vol. I, Academic Press, New York, 1967, pp. 759-784.

${ }^{24}$ Chodura, R. and Pohl, F., "Hydrodynamic Equations for Plasmas in Strong Magnetic Fields - II Transport Equations Including Collisions," Plasma Physics, Vol. 13, 1971, pp. 645-658.

${ }^{25}$ Demars, H. G. and Schunk, R. W., "Transport Equations for Multispecies Plasmas Based on Individual Bi-Maxwellian Distributions," Journal of Physics D: Applied Physics, Vol. 12, 1979, pp. 1051-1077.

${ }^{26}$ Hittinger, J. A., Foundations for the Generalization of the Godunov Method to Hyperbolic Systems with Stiff Relaxation Source Terms, Ph.D. thesis, University of Michigan, 2000.

${ }^{27}$ Kennard, E. H., Kinetic Theory of Gases, McGraw-Hill, New York, 1938.

${ }^{28}$ Barth, T. J., "Recent Developments in High Order K-Exact Reconstruction on Unstructured Meshes," Paper 93-0668, AIAA, January 1993.

${ }^{29}$ Venkatakrishnan, V., "On the Accuracy of Limiters and Convergence to Steady State Solutions," Paper 93-0880, AIAA, January 1993.

${ }^{30}$ Roe, P. L., "Approximate Riemann Solvers, Parameter Vectors, and Difference Schemes," Journal of Computational Physics, Vol. 43, 1981, pp. 357-372.

${ }^{31}$ Einfeldt, B., "On Godunov-Type Methods for Gas Dynamics," SIAM Journal on Numerical Analysis, Vol. 25, 1988, pp. 294-318. 
${ }^{32}$ Coirier, W. J. and Powell, K. G., "Solution-Adaptive Cartesian Cell Approach for Viscous and Inviscid Flows," AIAA Journal, Vol. 34, No. 5, May 1996, pp. 938-945.

${ }^{33}$ Sachdev, J. S. and Groth, C. P. T., "A Mesh Adjustment Scheme for Embedded Boundaries," Communications in Computational Physics, (Accepted for publication) 2007.

${ }^{34}$ Sachdev, J. S., Groth, C. P. T., and Gottlieb, J. J., "A Parallel Solution-Adaptive Scheme for Predicting Multi-Phase Core Flows in Solid Propellant Rocket Motors," International Journal of Computational Fluid Dynamics, Vol. 19, No. 2, 2005, pp. $157-175$.

${ }^{35}$ McDonald, J. G., Sachdev, J. S., and Groth, C. P. T., "Use of the Gaussian Moment Closure for the Modelling of Continuum and Micron-Scale Flows with Moving Boundaries," Proceedings of the 4th International Conference on Computational Fluid Dynamics, Ghent, Belgium, July 10-14, 2006.

${ }^{36}$ Coudeville, H., Trepaud, P., and Brun, E., "Drag Measurements in Slip and Transition Flow," Proceedings of the Fourth International Symposium on Rarefied Gas Dynamics, Academic Press, New York, 1965.

${ }^{37}$ Patterson, G. N., Introduction to the Kinetic Theory of Gas Flows, University of Toronto Press, Toronto, 1961. 2005.

${ }^{38}$ Suzuki, Y. and van Leer, B., "Application of the 10-moment model to MEMS flows," Paper 2005-1398, AIAA, January

${ }^{39}$ Allegre, J., Raffin, M., and Lengrand, J. C., "Experimental Flowfields Around NACA0012 Airfoils Located in Subsonic and Supersonic Rarefied Air Streams," Numerical Simulation of Compressible Navier-Stokes Flows, edited by M. O. Bristeau, R. Glowinski, J. Periaux, and H. Viviand, Vol. 18 of Notes on Numerical Fluid Dynamics, Fried. Vieweg and Sohn, Braunschweig, Germany, 1987, pp. 59-68. 